Rev. Elev. Méd. vét. Pays trop., 1973, 26 (2) : $249-59$

\title{
Modes d'exploitation des pâturages de Stylosanthes gracilis
}

\author{
par P. GRANIER $\left(^{*}\right)$
}

\begin{abstract}
RESUME
L'auteur a étudié l'influence de la hauteur et du rythme de l'exploitation de Stylosanthes gracilss sur les rendements en matière sèche et matières azotées. Les coupes hautes donnent des regains plus importants en saison sèche que les coupes basses et présentent plus d'intérêt puisque les rendements annuels sont sensiblement les mêmes pour chaque mode d'exploitation.

Stylosanthes gracilis a une croissance lente, les coupes basses le font régresser.

Son utilisation est étudiée pour chaque type d'exploitation (pâturage continu, réserves de foin).
\end{abstract}

\section{INTRODUCTION}

Le problème alimentaire en pays tropical à saison sèche marquée est caractérisé par le fait que les pâturages naturels sont composés essentiellement de graminées cespiteuses à port généralement élevé. A la fructification, ces graminées élaborent des tissus riches en hémicellulose et lignine, et le bétail, au cours de la saison sèche ne dispose plus que d'un aliment peu digestible, pauvre en matières azotées et dépourvu de carotènes. L'amélioration de ces pâturages est indispensable si l'on veut accrồtre la productivité de l'élevage.

Les travaux concernant le Stylosanthes gracilis $(3,4)$ ont montré que cette légumineuse présentait un très grand intérêt. Son implantation est très facile et économique (3) puisqu'il suffit d'un simple disquage du sol débarrassé ou non de la végétation naturelle par le feu. Une fois installé, la charge à l'hectare qu'il peut supporter est de l'ordre de $2 \mathrm{UBT} / \mathrm{ha}$

(*) Région de Recherches Vétérinaires et Zootechniques de Madagascar, B.P. $n^{\circ}$ 862, Tananarive, République Malgache.

Adresse actuelle : I.E.M.V.T., 10, rue Pierre Curie, 94700 Maisons-Alfort, France.
$(4,5)$ ce qui est une amélioration importante par rapport à la charge des savanes naturelles. L'expérimentation ayant démontré (5) qu'il résistait aux feux, au broutage, au piétinement, qu'il était appété par le bétail et qu'il améliorait les sols, il était normal qu'il soit adopté par les services chargés de la vulgarisation agricole et qu'il connaisse une large diffusion.

Une fois résolus les problèmes de son implantation et de son intégration dans l'assolement (5), se poseront à leur tour les problèmes concernant les modes d'exploitation possibles en fonction de la saison, du bétail, de la structure de l'exploitation, etc.

C'est pour répondre à ces questions que des expérimentations ont été effectuées au Centre de Recherches Zootechniques et Fourragères de Kianjasoa, situé au milieu d'une zone dans laquelle le Stylosanthes connaît actuellement une large expansion.

\section{ETUDE EXPERIMENTALE}

Les travaux ont été inspirés de ceux concernant l'exploitation de la luzerne (8) et de 
diverses graminées (7) en pays tempérés et qui montrent que la hauteur de coupe et la fréquence des coupes ont une influence sur les rendements annuels. Les résultats obtenus par les chercheurs diffèrent selon les espèces végétales étudiées, et l'approvisionnement des plantes en eau et sels minéraux.

\section{La hauteur de coupe}

On peut exploiter une plante en la coupant au ras du sol (coupe basse) ou bien à une certaine hauteur (coupe haute). Des essais préliminaires sur le Stylosanthes ont montré que:

1. Dans le cas d'une première coupe basse, on obtenait :

- un rendement élevé en matière sèche;

- un rendement élevé en matières azotées;

- des regains peu importants en saison sèche, mais riches en matières azotées.

2. En première coupe haute, si les rendements étaient moins élevés, par contre les regains étaient importants et la vitesse de repousse plus rapide que dans le cas d'une coupe basse, contrairement à ce que l'on observe sur les plantes des pays tempérés pendant la période de végétation active (printemps) dont les rendements sont accrus par des coupes basses.

Cette constatation est d'autant plus importante que le facteur limitant de la charge annuelle est représenté par la productivité des regains en saison sèche.

\section{Intervalle entre les coupes}

Au cours de ces essais, l'échelonnement des coupes montrait que dans tous les cas la vitesse de croissance du Stylosanthes était lente, et que les coupes tardives, de fin de saison des pluies, donnaient des regains nuls ou négligeables. A partir de ces constatations, un protocole expérimental a été mis en place pour déterminer les rendements saisonniers et annuels en matière sèche et matières azotées en fonction des hauteurs et des dates de coupe.

\section{Protocole expérimental}

Sur un pâturage homogène on a délimité deux objets :

- Coupe basse à $2 \mathrm{~cm}$ du sol (*).

(*) Cet objet reproduit les effets du surpâturage qui peut être une cause d'élimination du Stylosanthes.

\section{- Coupe haute à $15 \mathrm{~cm} \mathrm{du} \mathrm{sol.}$}

Sur chacun de ces objets, on échelonne les premières coupes en saison des pluies en se basant sur le stade végétatif :

- Coupe précoce en mars (stade feuillu);

- Coupe tardive en avril (stade ramifié);

- Coupe à la préfloraison au début du mois de mai;

- Coupe à la floraison à la fin du mois de de mai.

En fin de saison sèche (fin octobre), on coupe les regains à $2 \mathrm{~cm}$ du sol. Un témoin est laissé comme une réserve de saison sèche et exploité une seule fois en octobre.

Pour chaque coupe, on note:

- les rendements en vert;

- les rapports feuilles/tiges;

- les pourcentages de matière sèche (M.S.);

- les pourcentages de matières azotées totales (M.A.T.).

Dans le cadre de cette étude des essais de fanage ont été faits en mai afin de contrôler les résultats de divers modes de fanage sur la valeur du foin.

$$
\star \star
$$

Ces travaux ont été effectués pendant 3 années consécutives :

- 1969 : essais préliminaires;

- 1970: année à pluviométrie déficiente $(1.231 \mathrm{~mm})$;

- 1971: année à pluviométrie normale bien répartie $(1.576,3 \mathrm{~mm})$.

\section{RESULTATS}

\section{Productivité annuelle} et productivité de saison sèche

(tableaux $\mathrm{n}^{\text {os }} \mathrm{I}$, II, graphiques $\mathrm{n}^{\text {os }}$ I, II, III)

Dans le tableau $\mathrm{n}^{\circ}$ I nous donnons les résultats globaux obtenus par l'ensemble des coupes. Il apparaît :

- que, dans le cas des premières coupes basses, la dernière effectuée le 25 mai n'a pas donné de regains en saison sèche;

- que, dans le cas des premières coupes hau- 
TABLEAU $\mathrm{N}^{\circ} \mathrm{I}$

Productivité annuelle

(en $\mathrm{t} / \mathrm{ha}$ de M.S.; en $\mathrm{kg} / \mathrm{ha}$ de M.A.T.)

\begin{tabular}{|c|c|c|c|c|c|c|c|c|}
\hline \multirow{2}{*}{\multicolumn{3}{|c|}{ Mode d'exploitation }} & \multicolumn{2}{|c|}{ Première coupe } & \multicolumn{2}{|c|}{ Deuxième coupe } & \multicolumn{2}{|c|}{ Total annuel } \\
\hline & & & M.S. & M.A.T. & M.s. & M.A.T. & M.S. & M.A.T. \\
\hline \multirow[t]{4}{*}{ Coupes } & $\begin{array}{l}\text { basses } \\
\text { Prêcoce }\end{array}$ & (1) & 6,18 & 778 & 2,2 & 257 & 8,38 & 1.035 \\
\hline & Tardive & (2) & 8,85 & 1.017 & 1,7 & 166 & 10,5 & 1.183 \\
\hline & Préfloraison & (3) & 8,1 & 910 & 1,7 & 185 & 9,8 & 1.095 \\
\hline & Floraison & (4) & 7,4 & 665 & - & - & 7,4 & 665 \\
\hline \multicolumn{9}{|c|}{ Coupes hautes } \\
\hline & Précoce & (5) & 0,71 & 75 & 3,55 & 320 & 4,26 & 395 \\
\hline & Tardive & (6) & 3,5 & 476 & 3,72 & 290 & 7,22 & 766 \\
\hline & Préf loraison & (7) & 5,4 & 653 & 4,56 & 420 & 9,96 & 1.073 \\
\hline & Floraison & (B) & 6,9 & 765 & 4,25 & 310 & 11,15 & 1.075 \\
\hline $\begin{array}{l}\text { Coupe } \\
\text { saison }\end{array}$ & $\begin{array}{l}\text { unique en } \\
\text { n sèche }\end{array}$ & (9) & - & - & - & - & 6,0 & 335 \\
\hline
\end{tabular}

M.S. = Matière sèche; M.A.T. = Matière azotée totale.

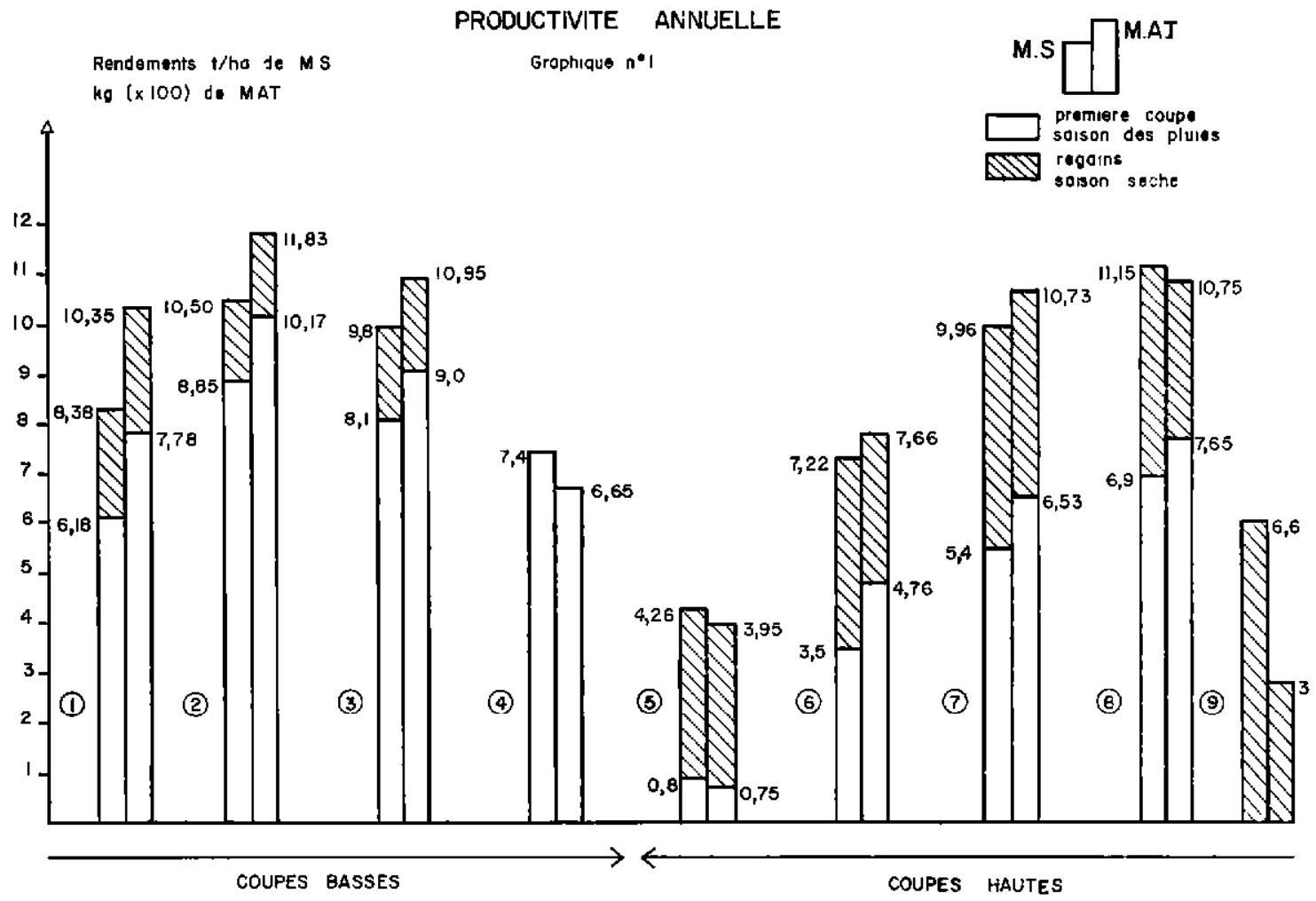

tes, la coupe précoce, écimant seulement la plante donne un rendement négligeable;

- que, la floraison du Stylosanthes étant très étalée dans le temps, puisque pratiquement on peut observer des fleurs depuis le mois de mai jusqu'en juillet, on n'obtient pas de résultats très différents entre les coupes effectuées à la préfloraison ou en pleine floraison. 
Afin de rendre plus compréhensible l'exposé des résultats, nous avons écarté, dans la discussion suivante, les premières coupes basses faites à la floraison (4) et les premières coupes hautes précoces (5) qui ne présentent pas d'intérêt pratique. De plus, étant donné que les résultats des coupes effectuées pendant la pé- riode de la floraison étaient très peu différents et que la mise à fleur est échelonnée, nous avons pris comme rendement la moyenne des coupes (2) et (3) d'une part, et (7) et (8) d'autre part.

Discussion (tableau $\mathrm{n}^{\circ}$ II et graphiques $\mathrm{n}^{\circ}$ II et III.

TABLEAU $\mathbb{N}^{\bullet}$ II

Productivité annuelle (moyennes) 1971

(en $\mathrm{t} / \mathrm{ha}$ de M.S.; en $\mathrm{kg} / \mathrm{ha}$ de M.A.T.)

\begin{tabular}{|c|c|c|c|c|c|c|}
\hline \multirow{2}{*}{ Mode d'exploitation } & \multicolumn{2}{|c|}{ Première coupe } & \multicolumn{2}{|c|}{ Deuxième coupe } & \multicolumn{2}{|c|}{ Total annuel } \\
\hline & M.S. & M.A.T. & M.S. & M.A.T. & M.S. & M.A.T. \\
\hline Caupes basses & & & & & & \\
\hline Prëcoce & 6,18 & 778 & 2,2 & 257 & 8,38 & 1.035 \\
\hline Prëfloraison (2) (3) & 8,4 & 963 & 1,7 & 175 & 10,1 & 1.138 \\
\hline Coupes bautes & & & & & & \\
\hline Tardive & 3,5 & 476 & 3,72 & 290 & 7,22 & 766 \\
\hline Préfloraison (7) (8) & 6,1 & 709 & 4,4 & 366 & 10,5 & 1.075 \\
\hline $\begin{array}{l}\text { Coupe unique } \\
\text { en saison sèche }\end{array}$ & - & - & 6 & 335 & 6 & 335 \\
\hline
\end{tabular}

PRODUCTIVITE ANNUELLE I97|

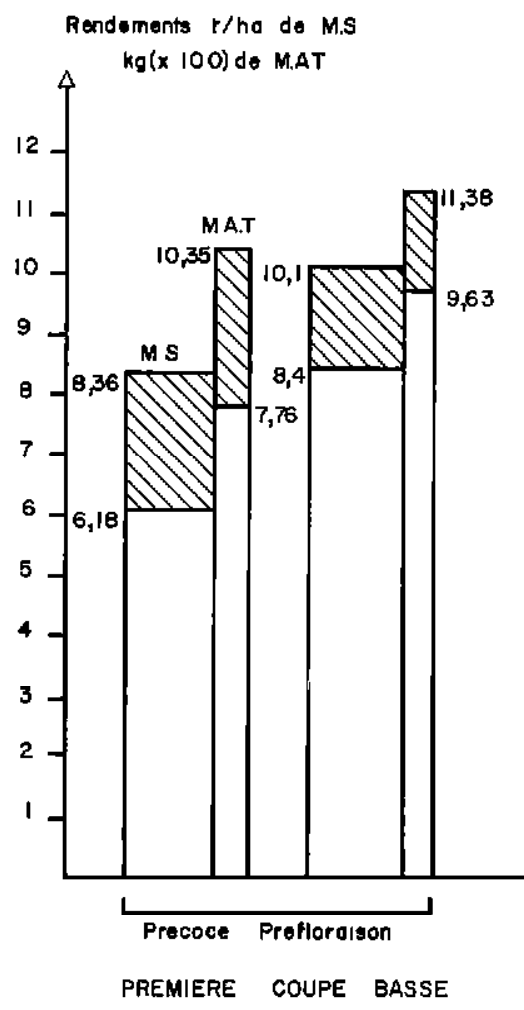

(moyennes)

grophique $n^{\circ 2}$

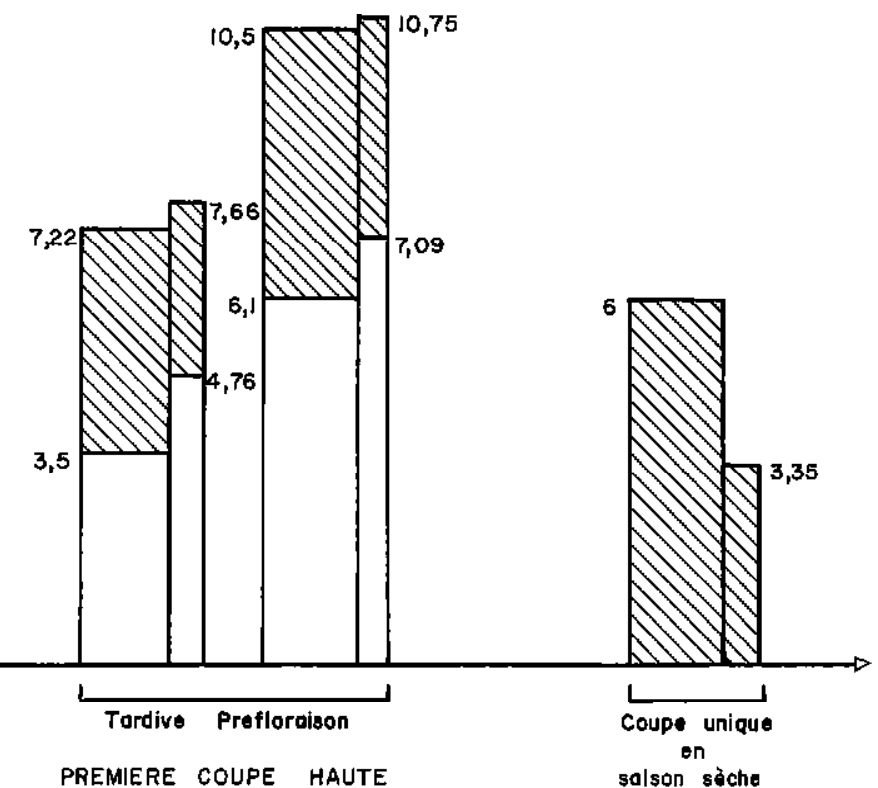


PRODUCTIVITE DES REGAINS

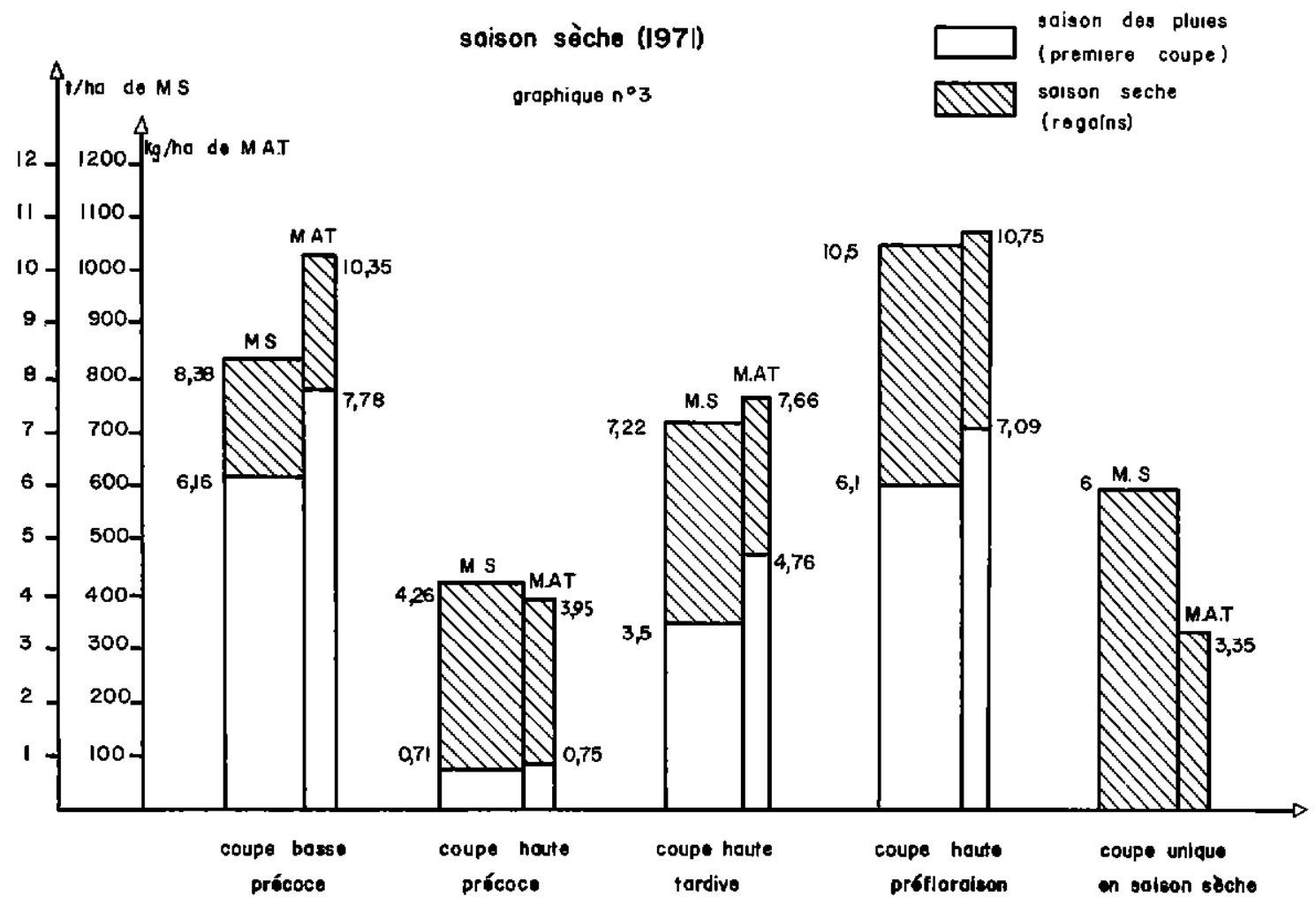

a) Pour chaque type de coupe, les rendements sont plus élevés sur les coupes effectuées à la préfloraison, ce qui confirme les données habituelles concernant les légumineuses.

b) Lorsqu'on exploite au ras du sol, une coupe précoce effectuée en pleine saison des pluies (6 mars) donne des regains plus élevés $(2,2 \mathrm{t} / \mathrm{ha}$ au lieu de 1,7$)$ et plus riches en matières azotées $(257 \mathrm{~kg} / \mathrm{ha}$ au lieu de 175) qu'une coupe à la préfloraison. L'allongement du temps de repos permet à la plante de donner des regains plus élevés, mais de toute façon, le rendement annuel est inférieur. Malgré l'enregistrement de pluies après la coupe $(150 \mathrm{~mm}$ environ), la productivité n'est pas stimulée par ce mode d'exploitation.

c) Ce sont les coupes hautes qui donnent les regains les plus importants en saison sèche et qui présentent le plus d'intérêt pour l'exploitant, puisque les rendements annuels sont sensiblement les mêmes pour chaque groupe.

Le Stylosanthes gracilis est une plante à croissance lente, à port normalement dressé et élevé : elle est déprimée par des coupes basses qui éliminent la majorité des points végétatifs sur les tiges et les feuilles qui, par photosynthèse, permettent à la plante de continuer sa croissance. Les réserves racinaires ne sont pas suffisantes pour provoquer le démarrage d'un nouveau cycle. Le fait que les tiges contiennent encore plus de 7 p. 100 de matières azotées (tableau $\mathbf{n}^{\circ}$ III) à la préfloraison, en mai, montre qu'elles doivent jouer un rôle dans la photosynthèse. Elles demeurent vertes pratiquement toute la saison sèche.

Dans le graphique $n^{\circ}$ III nous avons classé les coupes en considérant les rendements en saison sèche par ordre croissant. On remarque l'importance des coupes hautes, mais également la productivité du témoin qui, considéré comme une réserve sur pied de saison sèche, donne $6 \mathrm{t} / \mathrm{ha}$ de matière sèche en octobre. Il n'est pas sans intérêt de savoir que l'on peut ne pas exploiter la plante en saison des pluies et disposer d'une réserve d'une faible valeur nutritive mais appétible malgré tout pendant la période critique pour le bétail.

Les rendements annuels sont inférieurs à ceux obtenus avec deux coupes parce que la 
TABLEAU $\mathrm{N}^{\circ}$ III

Productivité comparêe. Feuilles et tiges

\begin{tabular}{|c|c|c|c|c|c|c|c|}
\hline \multirow[t]{2}{*}{ Coupes } & \multirow{2}{*}{$\begin{array}{c}\text { Rapport } \\
\text { Feuilles } \\
\text { Tiges } \\
\text { (M.S.) }\end{array}$} & \multicolumn{2}{|c|}{ M.S. (en p.100) } & \multicolumn{2}{|c|}{ M.A.T. (en p.100) } & \multicolumn{2}{|c|}{$\begin{array}{l}\text { Productivité des } \\
\text { feuilles en p. } 100 \\
\text { du total }\end{array}$} \\
\hline & & Feuilles & Tiges & Feuilles & Tiges & en M.S. & en M.A.T. \\
\hline Basses & & & & & & & \\
\hline Précoce & 1,15 & 24 & 26 & 16 & 9 & 51 & 65 \\
\hline Prêfloraison & 0,90 & 32 & 40 & 12,5 & 6,4 & 41 & 58 \\
\hline Hautes & & & & & & & \\
\hline Précoce & 2,5 & 26 & 20 & 14,5 & 9,0 & 71 & 80 \\
\hline Préfloraison & 1,1 & 31 & 35 & 14,4 & 7,5 & 52 & 67 \\
\hline
\end{tabular}

plante n'a pas été stimulée pendant la saison des pluies et parce qu'elle a perdu beaucoup de feuilles (mulch).

\section{Influence de la pluviométrie (graphique $\mathrm{n}^{\circ}$ IV)}

L'expérimentation s'est déroulée sur deux années consécutives très différentes sur le plan de la pluviométrie. Les rapports entre les divers rendements sont les mêmes et les conséquences pratiques à tirer de l'expérience sont donc confirmées par la répétition de 1971. L'influence de la pluviométrie, si elle ne modifie par l'ordre des résultats des différentes coupes, modifie très nettement les résultats quantitatifs, comme le montre le graphique $\mathrm{n}^{\circ}$ IV. Les rendements, en 1971, sont environ 1,4 fois plus élevés que ceux de 1970, pour tous les objets. Cela montre l'importance de la pluviométrie sur une plante à croissance lente, alors qu'une plante à croissance rapide effectue sa flambée de croissance presque indépendamment des pluies, comme nous l'avons déjà montré à propos des graminées tropicales (6). De plus, les résultats confirment qu'il n'y a épuisement de la plante dans aucun des cas.

\section{Rapport feuilles/tiges (tableau $\mathbf{n}^{\circ}$ III)}

L'étude des pourcentages des feuilles et des tiges montre (comme il est représenté sur le schéma ci-contre) que, dans les coupes hautes, l'écimage de la plante donne un pourcentage de feuilles élevé, alors que, dans les coupes basses, seules les précoces sont plus riches en feuilles qu'en tiges parce que, dès que les tiges s'élèvent, leur base se défolient.
Pour la fabrication de foin, on exploite en coupe basse :

- soit précoce pour obtenir le maximum de matières azotées;

- soit à la préfloraison si l'on recherche une réserve importante d'énergie.

Un foin fait avec une coupe haute serait plus digestible mais son prix de revient serait plus élevé parce que celui-ci augmente si les rendements en matière sèche diminuent, à cause de l'allongement des temps de travaux.

Ces données seront à prendre en considération si on parvient un jour à vulgariser la déshydratation des fourrages.
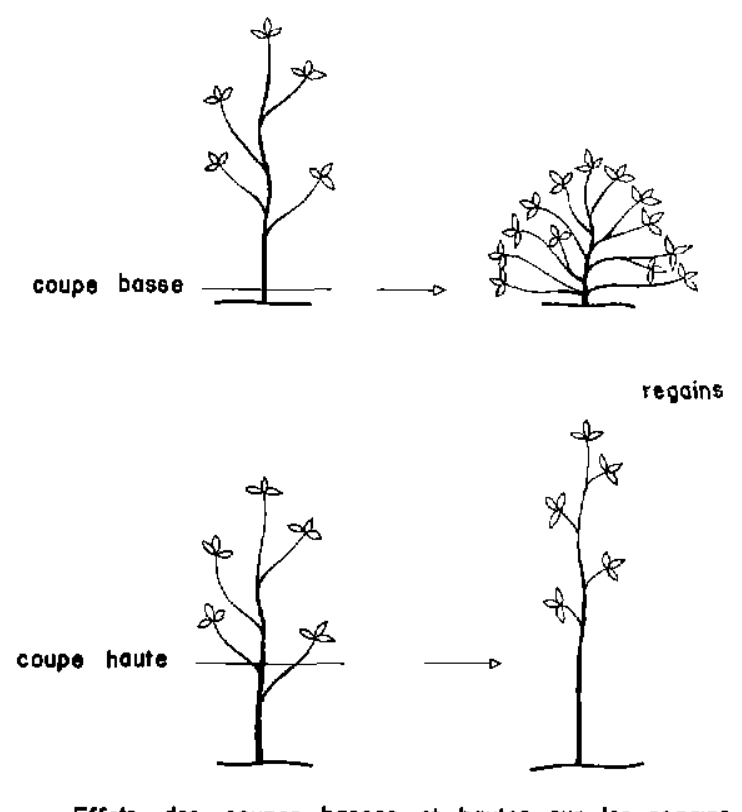

Effets des coupes bosses et hautes sur les regains 


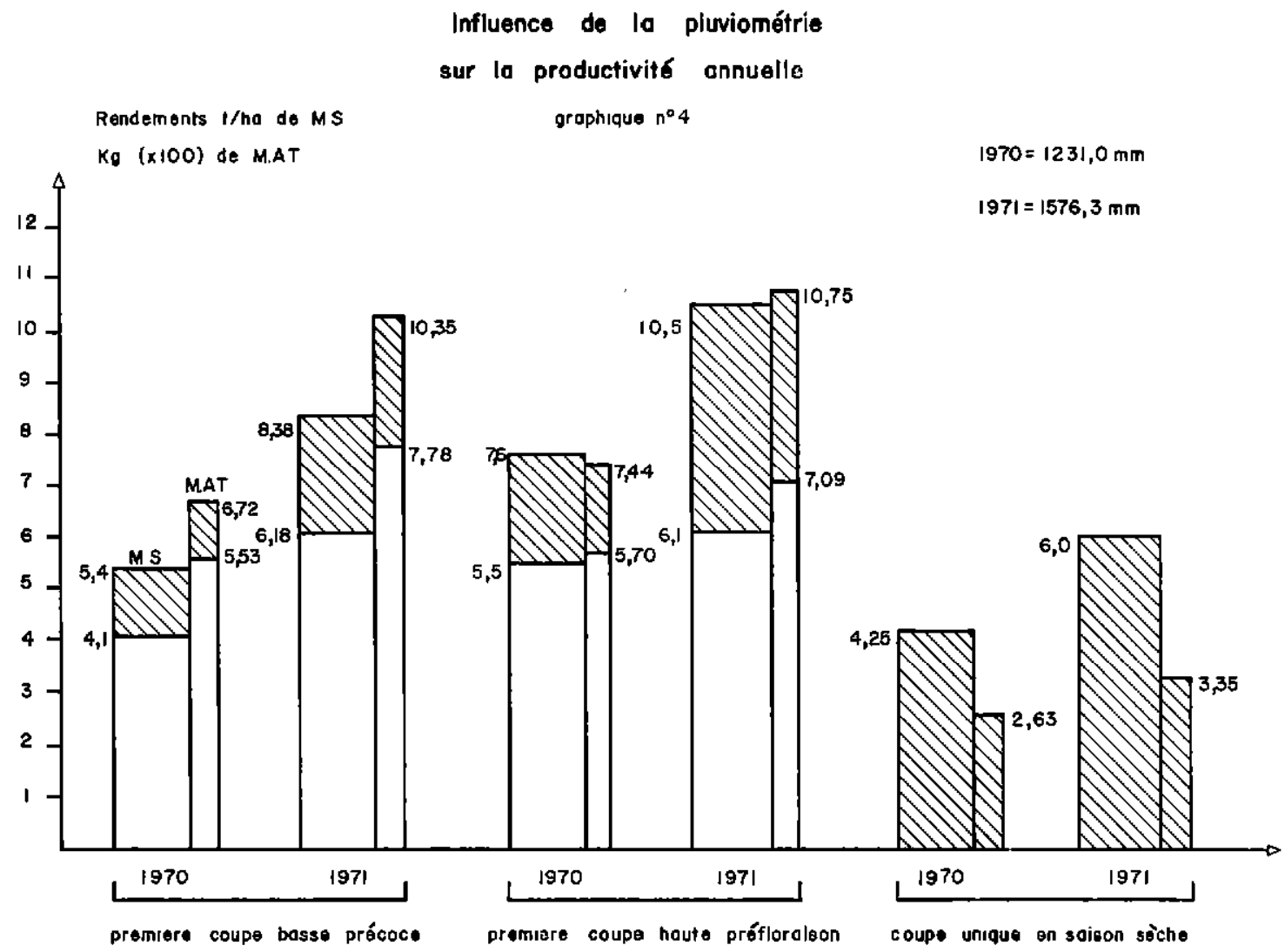

4. Productivité en Unités Fonrragères (U.F.)

Bien qu'il ne soit pas possible de préciser la valeur fourragère des échantillons sans déterminer la digestibilité et la transformation de l'énergie, nous donnons des résuitats en U.F. parce que, dans la pratique, les besoins du bétail étant définis en U.F., les charges à l'hectare et la production zootechnique ne peuvent être calculées qu'à partir des rendements en U.F. Nous avons attribué des valeurs moyennes très approximatives en nous basant sur les chiffres concernant la luzerne dans des conditions relativement voisines.

Le tableau $\mathrm{n}^{*}$ IV donne, par saison, la productivité comparée en U.F. des deux années 1970 et 1971 , qui, si l'on considère la moyenne

TABLEAU $\mathrm{N}^{\circ} \mathrm{IV}$

Productivité annuelle (moyennes)

(en U.F./ha (1))

\begin{tabular}{|c|c|c|c|c|c|c|c|}
\hline \multirow{2}{*}{ Mode d'exploitation } & \multicolumn{2}{|c|}{ Première coupe } & \multicolumn{2}{|c|}{ Deuxième coupe } & \multicolumn{3}{|c|}{ Total annue1 } \\
\hline & 1970 & 1971 & 1970 & 1971 & 1970 & 1971 & $\begin{array}{l}\text { Moyenne } \\
\text { arrondic }\end{array}$ \\
\hline \multicolumn{8}{|l|}{ Coupes basses } \\
\hline Précoce & 2.255 & 3.400 & 520 & 880 & 2.775 & 4.280 & 3.020 \\
\hline Préflaraison & 3.850 & 4.620 & 320 & 680 & 4.170 & 5.300 & 4.735 \\
\hline \multicolumn{8}{|l|}{ Coupes hautes } \\
\hline Précoce & 1.650 & 1.925 & 800 & 1.490 & 2.450 & 3.415 & 2.930 \\
\hline Préfloraison & 3.025 & 3.355 & 840 & 1.760 & 3.865 & 5.115 & 4.490 \\
\hline $\begin{array}{l}\text { Coupe unique } \\
\text { en saison sèche }\end{array}$ & - & - & 1.700 & 2.400 & 1.700 & 2.400 & 2.000 \\
\hline
\end{tabular}

(1) Valeur fourragère de la matière sèche : saison des pluies : 0,55 ; saison sèche : 0,40 . 
des deux rendements, s'étale de 2.930 U.F. à 4.735 U.F./an. Il apparaît que les coupes à la préfloraison sont les plus productives sur une année entière.

Si l'on considère la productivité en saison sèche on remarque que :

- les premières coupes basses précoces donnent les meilleurs résultats parce que le temps de repos est plus long;

- les premières coupes hautes donnent des résultats qui sont indépendants des dates et qu'il n'y a pas de proportionnalité entre les rendements de saison des pluies et ceux de saison sèche.

Dans la pratique, les rendements nets doivent tenir compte d'un pourcentage de refus de l'ordre de 25 p. 100 au pâturage en saison des pluies et 10 p. 100 en fin de saison sèche, si le broutage est intense.

\section{Rapport M.A.D./U.F.}

L'apport des matières azotées digestibles (M.A.D.) par Unité Fourragère (U.F.) est un critère qui permet de juger globalement la valeur d'un fourrage et d'apprécier le type de spéculation possible ainsi que la nécessité d'une complémentation.

En saison des pluies, par exemple, si nous attribuons une valeur de 0,55 U.F. $/ \mathrm{kg}$ de M.S. et une digestibilité de 65 p. 100 pour les matières azotées. Le rapport devient:

$$
\begin{aligned}
0,55 \times 6.180 \mathrm{~kg} & =3.400 \text { U.F. } \\
\frac{778 \mathrm{~kg} \times 65}{100} & =505 \mathrm{~kg} \text { M.A.D. } \\
\frac{\text { M.A.D. }}{\text { U.F. }} & =148
\end{aligned}
$$

En saison sèche, pour une valeur fourragère de 0,40 par $\mathrm{kg}$ de M.S. et un coefficient de digestibilité des matières azotées de l'ordre de 40 p. 100 seulement, on a :

$$
\begin{aligned}
0,40 \times 3.720 \mathrm{~kg} & =1.488 \text { U.F. } \\
\frac{290 \mathrm{~kg} \times 40}{100} & =116 \mathrm{~kg} \text { M.A.D. } \\
\frac{\text { M.A.D. }}{\text { U.F. }} & =77
\end{aligned}
$$

\section{Exploitation et charge à l'hectare}

La charge à l'hectare théorique que l'on peut déduire des résultats ci-dessus confirme la

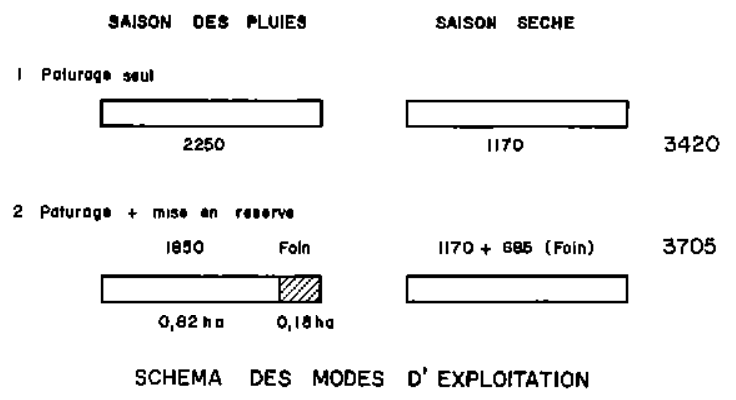

charge obtenue en fait dans les essais d'embouche sur Stylosanthes publiés par ailleurs (5) qui est de l'ordre de $2 \mathrm{UBT} / \mathrm{ha}$ (UBT = $250 \mathrm{~kg}$ ).

On peut envisager deux modes d'exploitation :

- pâturage seul;

- pâturage et mise en réserve de foin.

\section{a) Pâturage seul}

Le troupeau est laissé, toute l'année sur le pâturage. Nous considérons donc le cas d'une première coupe haute. Les rendements en U.F. disponibles, d'après le tableau $\mathrm{n}^{\circ} \mathrm{IV}$, en tenant compte des moyennes des deux années, des refus et du fait que le pâturage s'étale depuis la coupe précoce jusqu'à la floraison, sont de :

- Saison des pluies $=2.250$ U.F./ha;

- Saison sèche $\quad=1.170$ U.F./ha.

D'après les dates des coupes et la longueur des temps de repos, on peut admettre une répartition de 185 jours pour la première coupe et 180 jours pour celle des regains. La charge limitante de saison sèche est de:

$$
\frac{1.170 \text { U.F. }}{180}=6,5 \text { U.F./j }
$$

Il est donc possible de maintenir à l'entretien seulement 2 U.B.T./ha, les besoins étant de l'ordre de 2,8 U.F./j/tête. En saison des pluies, on aura un excédent disponible pour la croissance de :

$$
2.250-(185 \times 2,8 \times 2)=1.214 \text { U.F. } / \text { ha }
$$

Soit, si l'on admet un indice de consommation de l'ordre de 12 U.F./kg de gain, un gain global par tête de: $\frac{1.214}{12 \times 2} \# 50 \mathrm{~kg}$ par tête en saison des pluies. 
On peut dans la pratique comparer la productivité de la saison sèche avec une coupe unique en saison sèche, la croissance à cette saison étant faible, et compensée par la chute des feuilles les plus âgées.

\section{b) Pâturage + mise en réserve de foin}

En saison des pluies, on réserve une parcelle qui sera fauchée au ras du sol à la préfloraison. Le bétail pâture le reste. La distribution d'un supplément de foin en saison sèche permet de couvrir les besoins de la croissance puisque les besoins d'entretien sont assurés par le pâturage. Si nous estimons à 5 U.F./tểte/j ces besoins, nous avons la répartition suivante :

- Besoins en saison des pluies = 5 U.F. $\times 2 \times 185 \mathrm{j}=1.850$ U.F.

- Besoins en saison sèche $=$

5 U.F. $\times 2 \times 180 \mathrm{j}=1.800$ U.F.

Sur la partie pâturée, nous considérons les rendements obtenus en coupe haute soit 2.390 U.F./ha et, sur la partie fauchée, les rendements plus élevés obtenus avec la coupe basse (3.175 U.F./ha).

Les besoins en saison des pluies étant de 1.850 U.F. et les disponibilité de 2.250 , on n'utilisera en pâturage que :

$$
\frac{1 \times 1.850}{2.250}=0,82 \text { ha pour } 2 \text { têtes }
$$

Il sera possible de faucher:

$1-0,82=0,18$ ha

ce qui donnera une réserve de :

$$
\frac{3.810 \text { U.F. } \times 0,18}{1} \# 685 \text { U.F. }
$$

Ces 685 U.F. distribuées en supplément en saison sèche donnent une disponibilité totale pendant cette saison de :

$$
1.170+685=1.855 \text { U.F. }
$$

ce qui permettra de maintenir la même ration sur le plan énergétique toute l'année - et d'accroître le gain de poids annuel à environ $70 \mathrm{~kg} /$ tête.

Note : Les calculs concernant le premier cas (pâturage seul) ont été confirmés par l'expérimentation.

\section{APPLiCATIONS PRATIQUES ET CONCLUSION}

Les rézultats de l'expérimentation peuvent être résumés ainsi :

- la hauteur de la coupe a une influence sur les rendements en matière sèche et matières azotées totales;

- les coupes basses donnent des rendements supérieurs en saison des pluies;

- les coupes hautes donnent des regains plus élevés en saison sèche;

- les dates des coupes ont une influence sur les rendements, la période la plus favorable étant la préfloraison;

- le Stylosanthes a une croissance lente, l'allongement du temps de repos est favorable à un accroissement du rendement des regains. Il est déprimé par les coupes basses et ne doit être exploité que 2 fois/an, ou bien l'intégrité de l'axe principal lignifié doit être respectée (schéma $\mathrm{C}$ );

- les tiges semblent jouer un rôle important dans la photosynthèse et le développement de la plante.

\section{Conséquences}

La comparaison des résultats permet de préciser les divers modes d'exploitation possibles. On peut assimiler les coupes hautes à un pâturage rationné et les coupes basses à un fauchage mécanique. Le Stylosanthes, à cause de sa croissance lente, est une plante très plastique qui peut s'intégrer dans divers types d'exploitation.

C'est une plante qui se prête bien au pâturage et chaque fois qu'il est possible, c'est ce mode d'exploitation qu'il faut adopter. Mais la productivité saisonnière montre qu'on aura un excédent en saison des pluies et la valorisation de cet excédent implique la fabrication de foin sur une partie des surfaces. Si dans le cas de l'embouche on peut réduire le fanage, dans une exploitation laitière, la mise en réserve de l'herbe à l'optimum de sa valeur fourragère est préférable.

Il faut retenir, que le pâturage en saison des pluies, pour correspondre à une coupe haute, doit être moyen, un surpâturage réduirait la pousse des regains, et qu'une supplémentation azotée est nécessaire en fin de saison sèche, 
Retour au menu

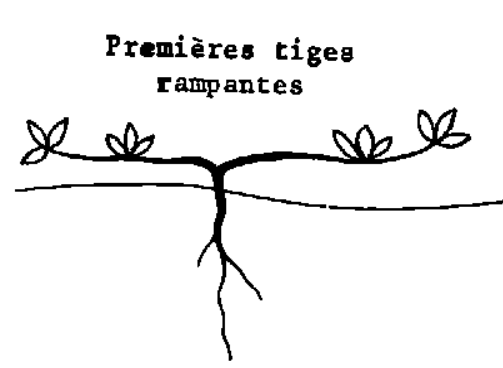

Exploitation moyenne rationnelle

STYLOSANTHES GRACILIS

$\mid$
N
D
$\mid$
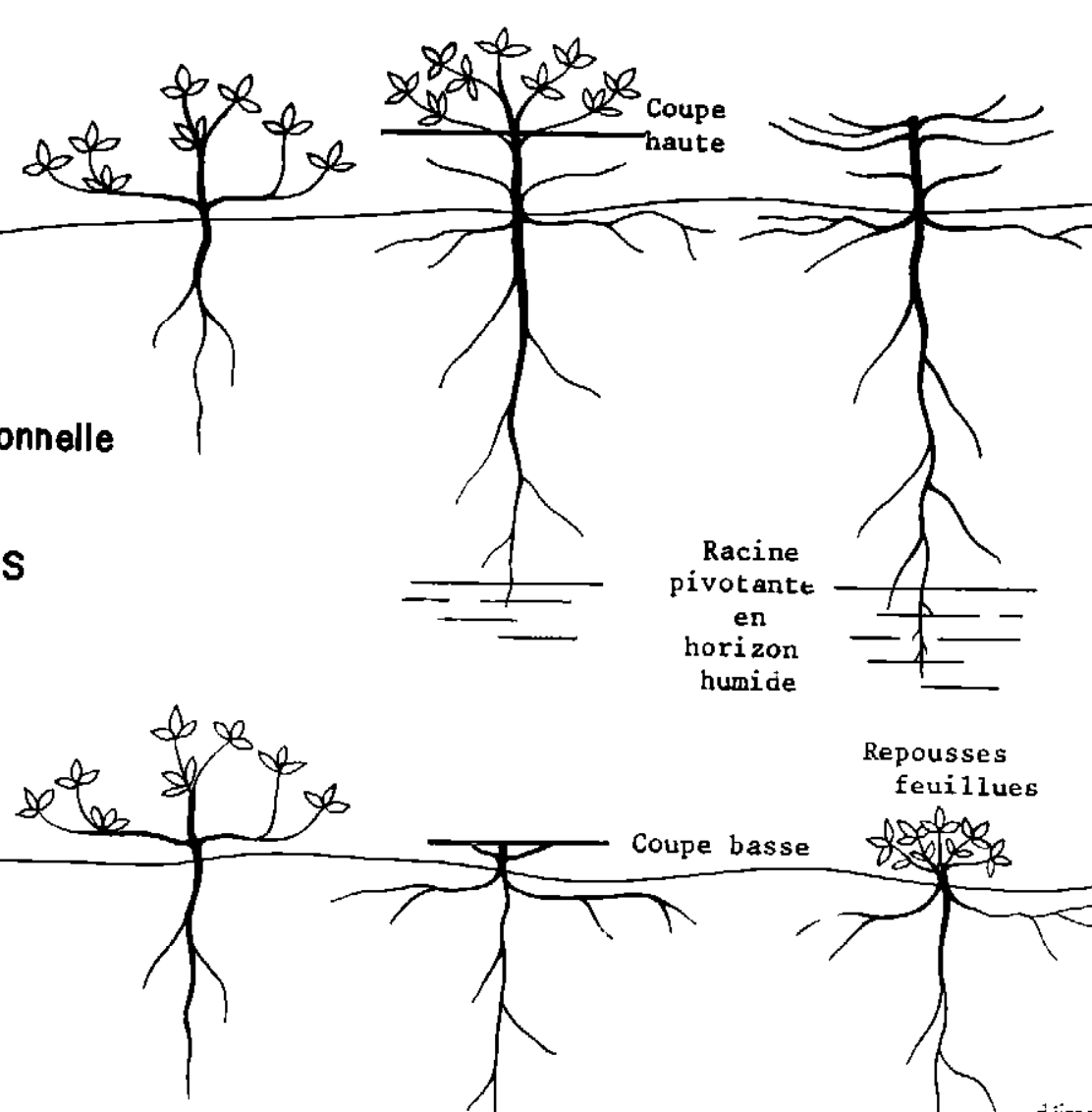

Surexploitation

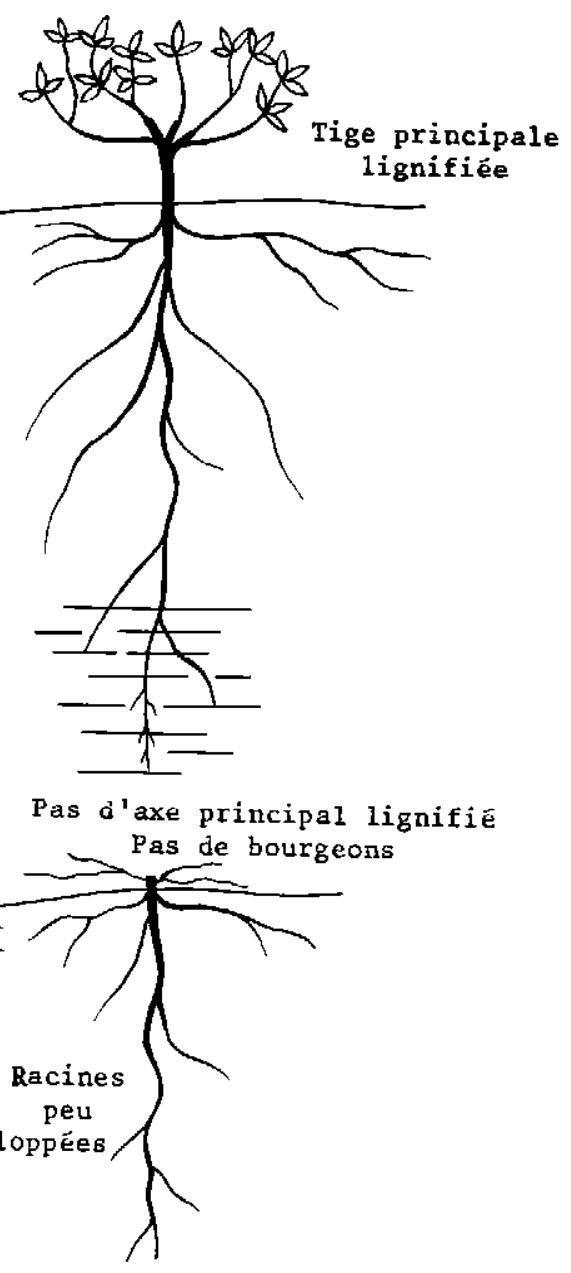

$\overline{\overline{-}}$

Schéma C 
l'apport de matières azotées digestibles étant insuffisant $\left(\frac{\text { M.A.D. }}{\text { U.F. }}=77\right)$ sauf pour du bétail adulte en finition.

Dans le cas où l'éleveur dispose encore de superficies importantes de pâturages naturels, il est possible de réserver le Stylosanthes pour la saison sèche, sa croissance lente permettant de disposer d'un fourrage sur pied, riche en énergie (mais insuffisant en matières azotées).

Si l'élevage s'intensifie, au contraire l'exploitation du Stylosanthes en pleine période de végétation active permettra de mettre en réserve un fourrage riche qui, après déshydratation fournira un aliment hautement énergétique pour l'embouche intensive ou la production laitière.

\section{SUMMARY}

Management of Stylosanthes gracilis

The author studies the influence of rythm and height of cuts on the yield in dry matter and crude protein of $S$. gracilis.

The cuts at higher level give greater dry-season aftermath than the lower and are more interesting since the annual yields are approximately the same in both cases.

$S$. gracilis grows slowly, low cuts tend to decrease its vigour.

Different types of management are then studied.

\section{RESUMEN}

Modos de explotación de los pastos de Stylosanthes gracilis

El autor estudió la influencia de la altura y del ritmo de la explotación de Stylosanthes gracilis sobre los rendimientos en materia seca y proteinas. Los cortes altos dan retoños más importantes durante la estación seca que los cortes bajos y tienen más interés puesto que los rendimientos anuales son casi los mismos en cada modo de explotación. Stylosanthes gracrlis tiene un crecimiento lento, los cortes bajos lo hacen retroceder.

Se estudia su utilización en cada tipo de explotación (pasto incontrolado, reservas de heno).

\section{BIBLIOGRAPHIE}

1. DEMARLY (Y.). La physiologie de la croissance en vert chez la luzerne. Bull, CET A, 1961, Etude no 542.

2. DEMARQUILLY (C.), WEISS (P.). Tableaux de la valeur alimentaire des fourrages. SE1, mars 1970 , Etude $\pi^{\circ} 42$.

3. GRANIER (P.), LAHORE (J.). Amélioration économique du pâturage naturel par le Stylosanthes gracilis. Madagascar, I.E.M.V.T., avril 1966.

4. GRANIER (P.), LAHORE (J.). Consommation d'herbe et charge à l'hectare avec le Stylosanthes gracilis. Madagascar, I.E.M.V.T., mars 1967.

5. GRANIER (P.). Le Stylosanthes gracilis à Madagascar Amélioration des savanes et intégration de l'élevage à l'agriculture. Bull. Madagascar, 1970 (289) : 522-550.

6. GRANIER (P.). Influence du mode d'exploitation sur les rendements du pâturage. Madagascar, I.E.M.V.T., juin 1970.

7. LAISSUS (R.). Importance de la hauteur de coupe sur le rendement et la composition d'une fléole. Fourrages, 1968 (35) : 27-38.

8. PLANCQUAERT (P.). Influence des stades de première coupe et des fréquences de coupes sur la production de la luzerne. Fourrages, 1968 (35): 6-14.

9. TULEY (P.). Stylosanthes gracilis. Herb. abstr., 1968, 38 (2): 87-94. 